\title{
Value of speckle-tracking echocardiography changes in monitoring myocardial dysfunction during treatment of sepsis: potential prognostic implications
}

\author{
Mariana de Braga Lima Carvalho Canesso $0^{1,2,5}$. Isabela Nascimento Borges ${ }^{2,5}$.

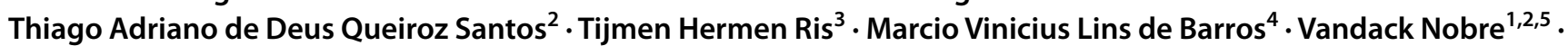 \\ Maria Carmo Pereira Nunes ${ }^{1,2,5}$
}

Received: 16 August 2018 / Accepted: 31 December 2018 / Published online: 7 March 2019

(c) Springer Nature B.V. 2019

\begin{abstract}
Speckle-tracking echocardiography (STE) has been increasingly used for detection of sepsis-related myocardial dysfunction. However, the impact of strain changes during sepsis treatment has not been defined. This study assessed STE at admission and during the treatment of patients with sepsis to evaluate its changes as a potential factor for predicting in-hospital outcome. This study included 26 patients with sepsis who underwent STE echocardiography on day 1 and 7 during treatment. Myocardial deformation of both ventricles was assessed using global longitudinal strain. The endpoint was in-hospital mortality. The mean age was $51.4 \pm 18.3$ years, and $54 \%$ were female. The average SOFA score at T0 was $8.6 \pm 3.8$ points and at day 7 was $4.9 \pm 4.7$ points. The left ventricular (LV) ejection fraction at baseline was $65.6 \pm 9.1 \%$, without changes in echocardiographic parameters during treatment. LV and RV longitudinal strain increased significantly in the patients who survived $(-18.8 \pm 3.6$ at $\mathrm{D} 1 \mathrm{vs}-20.8 \pm 2.5$ at $\mathrm{D} 7 ; \mathrm{p}=0.003$; and $-21.3 \pm 4.9$ at $\mathrm{D} 1 \mathrm{vs}-24.3 \pm 5.8$ at $\mathrm{D} 7 ; \mathrm{p}=0.035$, respectively), whereas strain values remained unchanged in those who died. After adjustment for the SOFA score, RV longitudinal strain at admission was associated with in-hospital mortality [adjusted odds ratio (OR) 0.760 ; 95\% confidence interval (CI) 0.591-0.977; p - 0.033]. STE improved significantly after the first week of treatment in patients with sepsis who survived compared with those patients who died during hospitalization. RV strain at admission predicted in-hospital mortality. An improvement in STE during sepsis treatment appears to be a useful tool for predicting in-hospital outcome.
\end{abstract}

Keywords Sepsis · Cardiovascular function · Speckle tracking echocardiography $\cdot$ Longitudinal strain $\cdot$ Ventricular function

Maria Carmo Pereira Nunes

mcarmo@waymail.com.br

1 Postgraduate Course of Infectious Diseases and Tropical Medicine, School of Medicine, Universidade Federal de Minas Gerais, Av. Professor Alfredo Balena, 190, Santa Efigênia, Belo Horizonte, Minas Gerais 30130100, Brazil

2 Hospital das Clinicas, School of Medicine, Universidade Federal de Minas Gerais, Belo Horizonte, MG, Brazil

3 Erasmus University Rotterdam -Erasmus MC, Rotterdam, The Netherlands

4 Mater Dei Hospital, Belo Horizonte, Brazil

5 Núcleo Interdiciplinar de Investigação em Medicina Intensiva (NIIMI), Belo Horizonte, Brazil

\section{Introduction}

Cardiovascular dysfunction is frequent in patients with sepsis, driven primarily by release of cytokines, mitochondrial dysfunction, and tissue hypoxia that leads to myocardial injury $[1,2]$. Impaired cardiovascular performance often present as new-onset left ventricular (LV) or right ventricular (RV) dysfunction by echocardiography, defined as septic cardiomyopathy (SCM). However, the impact of sepsis-related myocardial dysfunction on outcomes remains unclear. Some studies showed that cardiovascular dysfunction in sepsis is associated with worse hospital and longterm outcomes [3, 4], whereas other studies did not find this association $[2,5]$.

These controversial results can be related to the method used to assess ventricular contractility in the context of sepsis [6]. A meta-analysis including 14 studies failed to find 
any differences in LV or RV functions between the survivor and non-survivor patients with sepsis using conventional echocardiographic methods [7]. Nevertheless ventricular function in sepsis is often affected by several factors, especially changes in load conditions, which can influence the assessment of ventricular contractility by echocardiographic measurements [8].

Speckle-tracking echocardiography (STE) has been increasingly used for detection of early myocardial changes in sepsis, which may be not observed by conventional echocardiography [8-10]. Previous studies examining the value of STE in predicting mortality in sepsis have yielded conflicting results $[2,8,9,11,12]$. The reversible nature of myocardial dysfunction and illness severity difficult the evaluation of the prognostic value of STE in sepsis [10], and may contribute for this controversy. Therefore, strain changes in monitoring myocardial dysfunction during treatment would be useful to better predict outcome.

We hypothesized that STE technique can predict response to therapy and in-hospital outcome. Therefore, the aim of this study was to evaluate whether strain in patients with sepsis admitted to an intensive care unit (ICU) is a useful technique for monitoring therapy response and predicting in-hospital outcome.

\section{Methods}

We conducted a prospective observational study in the ICU of the University Hospital of the Universidade Federal de Minas Gerais (HC-UFMG), a mixed ICU with 18 beds. This study was approved by the institutional ethics committee, and informed consent was signed by all patients or their next of kin. To be included in the study the patients should have the diagnosis of sepsis [13] in the first $24 \mathrm{~h}$ of ICU admission. Patients who presented with LV systolic dysfunction at sepsis diagnosis, characterized by reduced LV ejection fraction or with other cardiac diseases were not included. The cases with limited image quality to measure STE were excluded after the echocardiogram.

Fifty-six patients were initially evaluated for potential eligibility, from which 30 were excluded (16 did not performed the second echocardiogram on day 7, 10 had associated cardiac disease, and 4 presented with tachyarrhythmia at admission), leaving 26 patients with all data available for analysis.

We collected clinical and laboratory data and performed echocardiographic studies on day 1 and 7 during hospitalization. Sequential Organ Failure Assessment (SOFA) score was calculated at ICU admission to quantifies the number and severity of organ dysfunction in six organ systems (respiratory, coagulatory, liver, cardiovascular, renal, and neurologic). The SOFA score is made of 6 variables, each representing an organ system, including $\mathrm{PaO}_{2} / \mathrm{FIO}_{2}$, platelets, bilirubin, mean arterial pressure, Glasgow coma score, and creatinine or urine output. Each organ system was assigned a point value from 0 (normal) to 4 (high degree of dysfunction).

Ventricular contractility was assessed by STE, as previously described [14-16] and global longitudinal strain was obtained from LV and RV. Serum samples were obtained from the blood, obtained for daily intensive care workups. The patients were followed until hospital discharge or death.

\section{Statistical analysis}

Clinical and echocardiographic data were analysed using the Statistical Package for Social Sciences (SPSS) version 22.1. Data were presented in absolute and relative frequencies, mean $\pm \mathrm{SD}$, median and interquartile range, as appropriate. The paired t-test (Student or Mann-Whitney) compared the continuous variables and the Chi square or Fisher's test compared the categorical variables.

Logistic regression analysis was performed to determine the potential value of STE in predicting in-hospital death. In the multivariate model, longitudinal strain of both LV and $\mathrm{RV}$ were included, after adjustment for SOFA score.

\section{Results}

The mean age was $51.4 \pm 18.3$ years, and $54 \%$ were female. Eleven patients (43\%) had a solid malignancy and three (12\%) suffered from chronic obstructive pulmonary disease (COPD). Septic shock was diagnosed in $88 \%$, with 17 patients $(65 \%)$ mechanically ventilated.

Table 1 summarizes the demographics, initial clinical and echocardiographic characteristics of the entire study population at $\mathrm{T} 0$ and after 1 week of treatment.

The average SOFA score at $\mathrm{T} 0$ was $8.6 \pm 3.8$ points and at day 7 was $4.9 \pm 4.7$ points in the entire population.

All patients had normal LV function assessed by ejection fraction at baseline $(65.6 \pm 9.1 \%)$, without changes in the conventional echocardiographic parameters with the treatment (Table 1). In the overall patients, there was an increase in longitudinal strain in both ventricles at the end of the first week of treatment (Table 1).

During hospitalization, seven patients (27\%) died. There were no differences related to comorbidities including solid malignancy, diabetes mellitus, hypertension, chronic kidney disease, or COPD between survivors and non-survivors. Baseline clinical characteristics of the patients according to in-hospital mortality are shown in Table 2. Patients who died had significantly higher SOFA score $(11.1 \pm 5$ vs $7.7 \pm 2.9$, 
Table 1 Clinical, laboratorial and echocardiographic data at baseline and after 7 days of treatment

\begin{tabular}{lccr}
\hline & Baseline & \multicolumn{1}{l}{ Time D7 } & p value \\
\hline SOFA (points) & $8.6 \pm 3.8$ & $4.9 \pm 4.7$ & $<0.001$ \\
Lactate levels $(\mathrm{mg} / \mathrm{dL})$ & $3.2 \pm 1.7$ & $1.5 \pm 0.9$ & $<0.001$ \\
Leukocytes $\left(\right.$ cells $\left.\times 10^{3} / \mu \mathrm{L}\right)$ & $16.5 \pm 10.7$ & $15.7 \pm 8.4$ & 0.701 \\
Platelets (cells $\left.\times 10^{3} / \mu \mathrm{L}\right)$ & $208.1 \pm 140.3$ & $291.2 \pm 187.2$ & 0.011 \\
C-reactive protein $(\mathrm{mg} / \mathrm{L})$ & $256.6 \pm 143.9$ & $135.0 \pm 106.3$ & 0.001 \\
Echocardiographic data & & & \\
LVEDD $(\mathrm{mm})$ & $45.1 \pm 3.9$ & $44.6 \pm 5.3$ & 0.538 \\
LVESD $(\mathrm{mm})$ & $29.0 \pm 4.2$ & $27.5 \pm 4.8$ & 0.051 \\
LVEF $(\%)$ & $65.6 \pm 9.1$ & $68.0 \pm 7.7$ & 0.132 \\
Global LV strain $(\%)$ & $-18.7 \pm 3.5$ & $-20.3 \pm 3.0$ & 0.024 \\
Global RV strain $(\%)$ & $-20.0 \pm 5.6$ & $-23.0 \pm 5.9$ & 0.012 \\
RV FAC $(\%)$ & $44.4 \pm 9.4$ & $48.8 \pm 7.1$ & 0.075 \\
TAPSE $(\mathrm{mm})$ & $19.9 \pm 3.9$ & $21.7 \pm 3.1$ & 0.063 \\
E velocity $(\mathrm{cm} / \mathrm{s})$ & $77.4 \pm 18.3$ & $70.5 \pm 17.7$ & 0.090 \\
A velocity $(\mathrm{cm} / \mathrm{s})$ & $64.0 \pm 16.8$ & $65.4 \pm 13.1$ & 0.560 \\
E/A ratio & $1.35 \pm 0.65$ & $1.15 \pm 0.45$ & 0.086 \\
E/e' ratio & $7.8 \pm 2.1$ & $6.7 \pm 1.6$ & 0.039 \\
\hline
\end{tabular}

Data presented as $\mathrm{n}(\%)$ or mean \pm standard deviation. $L V E D D$ left ventricular end-diastolic diameter, $L V E S D$ left ventricular end-systolic diameter, $L V E F$ left ventricular ejection fraction, $L V$ left ventricular, $R V$ right ventricular, $R V F A C$ right ventricular fractional area change, Tricuspid annular velocity tricuspid annular Doppler tissue velocity; A late mitral valve inflow velocity, TAPSE tricuspid annular plane systolic excursion, E/e' ratio $\mathrm{E} / \mathrm{e}^{\prime}$ ratio of early diastolic mitral inflow blood velocity to early diastolic mitral annular tissue velocity

$\mathrm{p}=0.036)$, arterial lactate levels $(4.1 \pm 1.7$ vs $2.6 \pm 1.5$,

Table 2 Baseline clinical characteristics of the patients according to in-hospital mortality

\begin{tabular}{llll}
\hline & Survivors & Deceased & p value \\
\hline Age (years) & $49.1 \pm 17.7$ & $57.6 \pm 19.8$ & 0.302 \\
Female (n/\%) & $11(58)$ & $3(43)$ & 0.495 \\
SOFA (points) & $7.7 \pm 2.9$ & $11.1 \pm 5$ & 0.036 \\
Lactate levels (mg/dL) & $2.6 \pm 1.5$ & $4.1 \pm 1.7$ & 0.040 \\
Urea (mg/dL) & $54.6 \pm 37.5$ & $92.9 \pm 16.8$ & 0.017 \\
Leukocytes (cells $\left.\times 10^{3} / \mu \mathrm{L}\right)$ & $15.1 \pm 11.1$ & $20.6 \pm 7.7$ & 0.175 \\
Platelets (cells $\left.\times 10^{3} / \mu \mathrm{L}\right)$ & $222.4 \pm 99.7$ & $183.7 \pm 222.2$ & 0.540 \\
C-reactive protein $(\mathrm{mg} / \mathrm{L})$ & $266.2 \pm 147.7$ & $316.0 \pm 140.6$ & 0.448 \\
LVEF $(\%)$ & $69.1 \pm 8.2$ & $64.3 \pm 9.3$ & 0.238 \\
RV FAC $(\%)$ & $44.5 \pm 10.6$ & $43.9 \pm 4.9$ & 0.874 \\
Global LV strain $(\%)$ & $-18.7 \pm 3.6$ & $-18.4 \pm 3.2$ & 0.803 \\
Global RV strain $(\%)$ & $-21.3 \pm 4.9$ & $-16.3 \pm 6.1$ & 0.042 \\
\hline
\end{tabular}

$\mathrm{p}=0.040)$ and urea $(92.9 \pm 16.8$ vs $54.6 \pm 37.5, \mathrm{p}=0.017)$ compared with those who survived.
STE according to in-hospital outcome showed a significant improvement in both ventricles after 7 days of treatment in the surviving compared to non-surviving patients. Left ventricular longitudinal strain in the patients who died was $-18.4 \pm 3.2 \%$ at D1 vs $-19.0 \pm 4.0$ at D7 $(\mathrm{p}=0.767)$ whereas in those who survived the strain was $-18.8 \pm 3.6$ at D1 vs $-20.8 \pm 2.5$ at D7 $(\mathrm{p}=0.003)$ (Fig. 1a). Similarly, right ventricular strain increased significantly in the patients who survived whereas in those who died the strain did not change during the treatment $(-16.3 \pm 6.1$ at D1 vs $-19.5 \pm 4.8$ at D7 in deceased; $\mathrm{p}=0.232$; and $-21.3 \pm 4.9$ at D1 vs $-24.3 \pm 5.8$ at D7 in survivors; $p=0.035$ ) (Fig. 1b).

After adjustment for the SOFA score, right ventricular global longitudinal strain at admission remained as an independent predictor of in-hospital mortality (adjusted OR $0.760 ; 95 \%$ CI 0.591-0.977; p - 0.033) (Table 3).
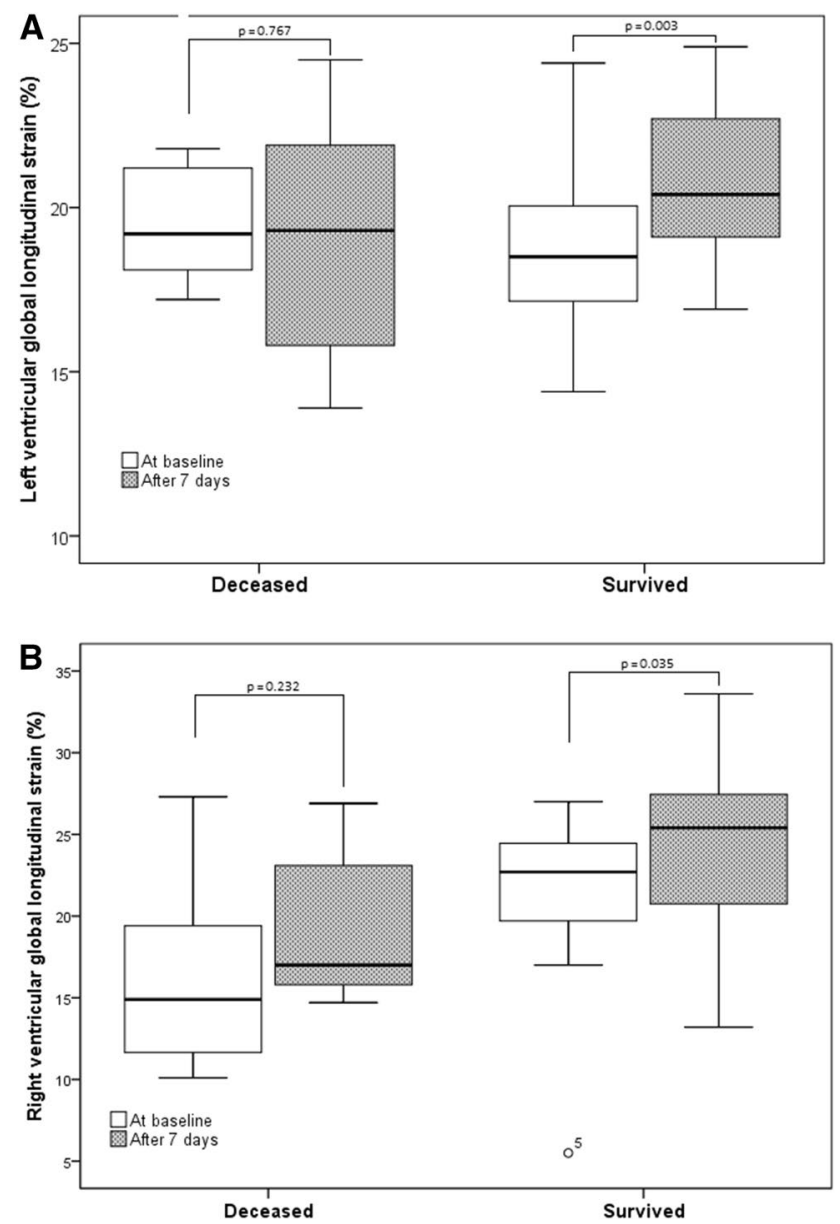

Fig. 1 a Left ventricular longitudinal STE of $-18.4 \pm 3.2 \%$ at D1 versus $-19.0 \pm 4.0$ at D7 in deceased patients; $\mathrm{p}=0.767$; and $-18.8 \pm 3.6$ at $D 1$ versus $-20.8 \pm 2.5$ at $D 7$ in survivors; $p=0.003$. b Right ventricular longitudinal STE of -14.9 [11.1/20.5] at D1 versus $-19.5 \pm 4.8$ at D7 in deceased; $p=0.232$; and -22.7 [19.7/24.6] at D1 versus $-24.3 \pm 5.8$ at D7 in survivors; $p=0.035$ 
Table 3 Predictors of in-hospital mortality obtained at $24 \mathrm{~h}$ of admission

\begin{tabular}{llll}
\hline & Odds ratio & $95 \%$ CI & p value \\
\hline SOFA (points) & 1.120 & $0.816-1.538$ & 0.483 \\
Global RV strain $(\%)$ & 0.760 & $0.591-0.977$ & 0.033 \\
\hline
\end{tabular}

\section{Discussion}

In the patient's population admitted in ICU with sepsis and preserved LV ejection fraction, an improvement in ventricular longitudinal strain was associated with a favorable prognosis. STE increased after the first week of treatment in the alive patients compared with those who died during hospitalization. Right ventricular global longitudinal strain at ICU admission was associated with in-hospital mortality. Global longitudinal strain changes might be useful for in-hospital risk stratification in sepsis.

Several studies corroborate the findings that strain detects ventricular dysfunction in septic patients with better sensitivity than conventional echocardiography [17-19]. STE is able to unmask systolic dysfunction, which may be missed when using conventional echocardiography. Additionally, STE may be useful in monitoring sepsis-induced myocardial dysfunction $[8,10,11,20]$, with potential impact on prognosis in patients with sepsis.

Studies addressing the prognostic value of STE in sepsis demonstrated controversial results [8, 10, 12]. Orde et al. assessing myocardial function by strain showed that LV dysfunction did not predict survival, whereas RV dysfunction was associated with high mortality in patients with severe sepsis or septic shock [8], which is agreement with our study. In contrast, Palmieri et al. demonstrated that global LV longitudinal strain was associated with mortality at 7-day follow-up [11]. A retrospective study found that LV and RV systolic functions are impaired in patients with early septic shock and preserved LV ejection fraction compared to major trauma patients [9].

Different from previous studies that evaluated the strain only at baseline $[3,8,17]$ our study measured strain in two consecutive moments. As myocardial dysfunction in patients with sepsis is usually reversible, we examined the impact of STE changes during treatment on in-hospital mortality. Interesting, an improvement of ventricular strain was associated with favorable outcome. A recent study showed that acute LV dysfunction was not associated with a worse prognosis in septic shock [2]. However, different from our study that used strain, this previous study assessed LV function by conventional measurement of LV ejection fraction [2].

In our present study, all patients with myocardial impairment detected by conventional echocardiographic assessment at sepsis diagnosis were excluded to avoid introducing confounding factors that may be associated with outcome.

The main limitation of the present study was the small number of patients included, and the results need to be confirmed by further studies.

\section{Conclusions}

Speckle-tracking ecocardiography improved significantly after the first week of treatment in patients with sepsis who survived compared with those patients who died during hospitalization. Right ventricular longitudinal strain at ICU admission was associated with in-hospital mortality. An improvement in biventricular longitudinal strain during sepsis treatment may predict a good in-hospital outcome.

Funding CNPq (Brazilian Council for Scientific and Technological Development) partly supported the study.

\section{Compliance with ethical standards}

Conflict of interest All authors declare that they have no conflict of interest.

\section{References}

1. Suffredini AF, Fromm RE, Parker MM, Brenner M, Kovacs JA, Wesley RA et al (1989) The cardiovascular response of normal humans to the administration of endotoxin. N Engl J Med 321:280-287

2. Vallabhajosyula S, Jentzer JC, Geske JB, Kumar M, Sakhuja A, Singhal A et al (2018) New-onset heart failure and mortality in hospital survivors of sepsis-related left ventricular dysfunction. Shock 49:144-149

3. Vallabhajosyula S, Kumar M, Pandompatam G, Sakhuja A, Kashyap R, Kashani K et al (2017) Prognostic impact of isolated right ventricular dysfunction in sepsis and septic shock: an 8-year historical cohort study. Ann Intensiv Care 7:94

4. Vallabhajosyula S, Sakhuja A, Geske JB, Kumar M, Poterucha JT, Kashyap R et al (2017) Role of admission troponin-T and serial troponin-T testing in predicting outcomes in severe sepsis and septic shock. J Am Heart Assoc 6

5. Pulido JN, Afessa B, Masaki M, Yuasa T, Gillespie S, Herasevich $\mathrm{V}$ et al (2012) Clinical spectrum, frequency, and significance of myocardial dysfunction in severe sepsis and septic shock. Mayo Clinic Proc 87:620-628

6. Kimchi A, Ellrodt AG, Berman DS, Riedinger MS, Swan HJ, Murata GH (1984) Right ventricular performance in septic shock: a combined radionuclide and hemodynamic study. J Am Coll Cardiol 4:945-951

7. Huang SJ, Nalos M, McLean AS (2013) Is early ventricular dysfunction or dilatation associated with lower mortality rate in adult severe sepsis and septic shock? A meta-analysis. Crit Care 17:R96

8. Orde SR, Pulido JN, Masaki M, Gillespie S, Spoon JN, Kane GC et al (2014) Outcome prediction in sepsis: speckle tracking echocardiography based assessment of myocardial function. Crit Care 18:R149 
9. Dalla K, Hallman C, Bech-Hanssen O, Haney M, Ricksten SE (2015) Strain echocardiography identifies impaired longitudinal systolic function in patients with septic shock and preserved ejection fraction. Cardiovasc Ultrasound 13:30

10. Ng PY, Sin WC, Ng AK, Chan WM (2016) Speckle tracking echocardiography in patients with septic shock: a case control study (SPECKSS). Crit Care 20:145

11. Palmieri V, Innocenti F, Guzzo A, Guerrini E, Vignaroli D, Pini $R$ (2015) Left ventricular systolic longitudinal function as predictor of outcome in patients with sepsis. Circ Cardiovasc Imaging 8:e003865; discussion e

12. Vallabhajosyula S, Rayes HA, Sakhuja A, Murad MH, Geske JB, Jentzer JC (2018) Global longitudinal strain using speckletracking echocardiography as a mortality predictor in sepsis: a systematic review. J Intensiv Care Med 885066618761750

13. Singer M, Deutschman CS, Seymour CW, Shankar-Hari M, Annane D, Bauer $M$ et al (2016) The third international consensus definitions for sepsis and septic shock (Sepsis-3). JAMA 315:801-810

14. Lang RM, Badano LP, Mor-Avi V, Afilalo J, Armstrong A, Ernande L et al (2015) Recommendations for cardiac chamber quantification by echocardiography in adults: an update from the American Society of Echocardiography and the European Association of Cardiovascular Imaging. Eur Heart J Cardiovasc Imaging 16:233-270
15. Collier P, Phelan D, Klein A (2017) A test in context: myocardial strain measured by speckle-tracking echocardiography. J Am Coll Cardiol 69:1043-1056

16. Morris DA, Krisper M, Nakatani S, Kohncke C, Otsuji Y, Belyavskiy E et al (2017) Normal range and usefulness of right ventricular systolic strain to detect subtle right ventricular systolic abnormalities in patients with heart failure: a multicentre study. Eur Heart J Cardiovasc Imaging 18:212-223

17. Chang WT, Lee WH, Lee WT, Chen PS, Su YR, Liu PY et al (2015) Left ventricular global longitudinal strain is independently associated with mortality in septic shock patients. Intensive Care Med 41:1791-1799

18. Shahul S, Gulati G, Hacker MR, Mahmood F, Canelli R, Nizamuddin $\mathbf{J}$ et al (2015) Detection of myocardial dysfunction in septic shock: a speckle-tracking echocardiography study. Anesth Analg 121:1547-1554

19. Innocenti F, Palmieri V, Guzzo A, Stefanone VT, Donnini C, Pini $\mathrm{R}$ (2018) SOFA score and left ventricular systolic function as predictors of short-term outcome in patients with sepsis. Intern Emerg Med 13:51-58

20. Zaky A, Gill EA, Lin CP, Paul CP, Bendjelid K, Treggiari MM (2016) Characteristics of sepsis-induced cardiac dysfunction using speckle-tracking echocardiography: a feasibility study. Anaesth Intensive Care 44:65-76 